\title{
Rationale and Design of the Hamburg City Health Study
}

\author{
Annika Jagodzinski ${ }^{1,2,3}$ (D) Christoffer Johansen ${ }^{3,4,5,6} \cdot$ Uwe Koch-Gromus $^{7} \cdot$ Ghazal Aarabi $^{8} \cdot$ Gerhard Adam $^{9}$. \\ Sven Anders ${ }^{10}$. Matthias Augustin ${ }^{11}$ - Ramona B. der Kellen ${ }^{3}$. Thomas Beikler ${ }^{12}$. Christian-Alexander Behrendt ${ }^{13}$. \\ Christian S. Betz ${ }^{14}$. Carsten Bokemeyer ${ }^{15} \cdot$ Katrin Borof $^{3}$. Peer Briken ${ }^{16}$. Chia-Jung Busch ${ }^{14}$. Christian Büchel ${ }^{17}$. \\ Stefanie Brassen ${ }^{17}$. Eike S. Debus ${ }^{13} \cdot$ Larissa Eggers $^{3}$. Jens Fiehler ${ }^{18}$. Jürgen Gallinat ${ }^{19} \cdot$ Simone Gellißen $^{18}$. \\ Christian Gerloff $^{20}$. Evaldas Girdauskas ${ }^{21}$ - Martin Gosau ${ }^{22}$ - Markus Graefen ${ }^{23}$. Martin Härter ${ }^{24} \cdot$ Volker Harth $^{25}$. \\ Christoph Heidemann ${ }^{3}$. Guido Heydecke ${ }^{8}$. Tobias B. Huber ${ }^{26}$. Yassin Hussein ${ }^{3}$. Marvin O. Kampf ${ }^{3}$. \\ Olaf von dem Knesebeck ${ }^{27}$. Alexander Konnopka ${ }^{28} \cdot$ Hans-Helmut König ${ }^{28} \cdot$ Robert Kromer $^{29}$. \\ Christian Kubisch $^{30}$. Simone Kühn ${ }^{19}$. Sonja Loges ${ }^{15,34} \cdot$ Bernd Löwe $^{31}$. Gunnar Lund ${ }^{9}$. Christian Meyer,32 . \\ Lina Nagel ${ }^{3} \cdot$ Albert Nienhaus $^{33} \cdot$ Klaus Pantel $^{34} \cdot$ Elina Petersen $^{3} \cdot$ Klaus Püschel $^{10} \cdot$ Hermann Reichenspurner $^{21}$. \\ Guido Sauter $^{35} \cdot$ Martin Scherer $^{36} \cdot$ Katharina Scherschel $^{2,32} \cdot$ Ulrich Schiffner $^{12} \cdot$ Renate B. Schnabel $^{1,2}$. \\ Holger Schulz ${ }^{24}$. Ralf Smeets ${ }^{22}$. Vladislavs Sokalskis ${ }^{3} \cdot$ Martin S. Spitzer ${ }^{29} \cdot$ Claudia Terschüren $^{25} \cdot$ Imke Thederan $^{23}$. \\ Tom Thoma ${ }^{3} \cdot$ Götz Thomalla $^{20} \cdot$ Benjamin Waschki $^{1,2,38} \cdot$ Karl Wegscheider $^{6} \cdot$ Jan-Per Wenzel ${ }^{1,3} \cdot$ Susanne Wiese $^{3}$. \\ Birgit-Christiane Zyriax ${ }^{2,37} \cdot$ Tanja Zeller ${ }^{1,2,3} \cdot$ Stefan Blankenberg ${ }^{1,2}$
}

Received: 16 August 2019 / Accepted: 31 October 2019 / Published online: 8 November 2019

(c) The Author(s) 2019

\begin{abstract}
The Hamburg City Health Study (HCHS) is a large, prospective, long-term, population-based cohort study and a unique research platform and network to obtain substantial knowledge about several important risk and prognostic factors in major chronic diseases. A random sample of 45,000 participants between 45 and 74 years of age from the general population of Hamburg, Germany, are taking part in an extensive baseline assessment at one dedicated study center. Participants undergo 13 validated and 5 novel examinations primarily targeting major organ system function and structures including extensive imaging examinations. The protocol includes validate self-reports via questionnaires regarding lifestyle and environmental conditions, dietary habits, physical condition and activity, sexual dysfunction, professional life, psychosocial context and burden, quality of life, digital media use, occupational, medical and family history as well as healthcare utilization. The assessment is completed by genomic and proteomic characterization. Beyond the identification of classical risk factors for major chronic diseases and survivorship, the core intention is to gather valid prevalence and incidence, and to develop complex models predicting health outcomes based on a multitude of examination data, imaging, biomarker, psychosocial and behavioral assessments. Participants at risk for coronary artery disease, atrial fibrillation, heart failure, stroke and dementia are invited for a visit to conduct an additional MRI examination of either heart or brain. Endpoint assessment of the overall sample will be completed through repeated follow-up examinations and surveys as well as related individual routine data from involved health and pension insurances. The study is targeting the complex relationship between biologic and psychosocial risk and resilience factors, chronic disease, health care use, survivorship and health as well as favorable and bad prognosis within a unique, large-scale long-term assessment with the perspective of further examinations after 6 years in a representative European metropolitan population.
\end{abstract}

Electronic supplementary material The online version of this article (https://doi.org/10.1007/s10654-019-00577-4) contains supplementary material, which is available to authorized users.

Extended author information available on the last page of the article 
Keywords Epidemiology · Prospective cohort study · Risk factors · Prevention · Coronary heart disease $\cdot$ Stroke $\cdot$ Dementia $\cdot$ Cancer $\cdot$ Health care $\cdot$ Vascular diseases $\cdot$ Oral health $\cdot$ Psychiatric and psychosomatic disorders $\cdot$ Ocular diseases $\cdot$ Respiratory diseases $\cdot$ Obesity $\cdot$ Nutrition $\cdot$ Lifestyle $\cdot$ Sexual dysfunction $\cdot$ Survivorship $\cdot$ Resilience $\cdot$ MRI imaging $\cdot$ Cardiac MRI $\cdot$ Brain MRI $\cdot$ Health service research $\cdot$ Hamburg

\section{Introduction}

Within the last decades, a change in the disease pattern has been observed. Today, the majority of the ageing populations in industrialized parts of the world will survive acute events and move into survivorship living with one or more of the following conditions, e.g., cardiovascular and neurovascular disease, cancer, respiratory diseases, diabetes [1]. This change is mostly explained by better diagnostics leading to identification of disease at an earlier stage and to the development of more effective treatments of these diseases.

In addition to the change in disease patterns a change in diagnostic abilities and personalized treatment opportunities has occurred. The integration of imaging, molecular biology and clinical information holds promise to better detect at risk individuals and to personalize treatment.

Besides this new perspective of living for years at risk or in treatment for a chronic health condition, this may also influence physical, mental and sexual health, quality of life, general disability and society's health expenses. In Germany, like in other affluent industrialized countries, the above mentioned chronic disease epidemic accounts for more than a third of the annual health expenditures, almost half of the total hospital payments [2]. It was estimated in 2013 , that 500,000 potentially productive life years were lost due to premature death by these conditions in the working-age population (29-59 years of age [3]).

Observational studies have changed practice of medicine and lifestyle for millions of people [4], e.g., the Framingham Heart Study, which for the first time identified risk factors for cardiovascular events [4]. The conditions for those who actually survived these diseases were only a minor part of the agenda. Thus, we established a prospective observational cohort study that can address the impact of information from biological samples, medical examinations and imaging, classic self-reported questionnaire data and their interplay on our understanding of common disease development. Issues of disease development, pathophysiological understanding, artificial intelligence and survivorship constitute the cornerstones of the Hamburg City Health Study.

Moreover, we establish a biobank including a wide variety of biomaterials enabling molecular analyses, that-in total, will lead to a better understanding of health, disease and survivorship.

\section{Objectives}

The Hamburg City Health Study has established a unique research platform with multiple risk assessment, numerous outcomes and imaging examinations in all participants, a sophisticated biobank and interdisciplinary network to address a wide range of questions about more than 30 major chronic diseases (see Box 1 and Fig. 1) and survivorship.

Therefore, the primary aims of the HCHS are to investigate in detail:

- the causes for the development of functional health impairments and major chronic diseases,

- the prognostic factors for surviving chronic diseases and

- identification of factors supporting life in survivorship of major chronic diseases.

\section{Study design and methods}

Hamburg is the second largest city in Germany with $1,830,584$ million inhabitants (31.12.2017 [5] from all social classes living in 7 districts and 104 urban quarters. The city is mostly urban, but has also some rural areas and a large harbor contributing to the environmental exposures of the population. 839,389 persons are older than 45 years and in 2017 approximately 90,000 persons moved away. On an annual basis a sample census is carried out mapping social conditions of the German population including a random sample of $1 \%$ of the whole population. As shown in Box 2 the Hamburg population in comparison to the German population is characterized by $4 \%$ more other nationalities, people are $7 \%$ more often single, $14 \%$ have a higher education and a higher income.

A total of 45,000 inhabitants, aged $45-74$ years are to be included, identified by a random sample from the official inhabitant data file divided into six age and gender strata. The HCHS is a joint interdisciplinary endeavor of physicians and scientists from the University Medical Center Hamburg-Eppendorf. Over 30 departments and institutes from the University Medical Center HamburgEppendorf work together in a unique cooperation at a single study center. 
Box 1 Overview of the main outcomes: the Hamburg City Health Study

Main outcome

Coronary artery disease and myocardial infarction

Atrial fibrillation

Heart failure

Dementia

Stroke

Cancer such as prostate cancer and skin cancer

Chronic kidney diseases

Arterial hypertension, hypercholesteremia and valvular diseases such as bicuspide aortic valve disease

Migraine

Musculoskeletal diseases such as osteoporosis and bone metastasis

Ocular diseases such as glaucoma, macular degeneration, fundus hypertonicus, retinal vessel disease and neoplasm

Oral health including periodontal disease and caries, oropharyngeal cancer and human papillomavirus (HPV)-infection

Psychiatric and psychosomatic disorders such as mental disorder or late - last depression

Pulmonary diseases such as chronic obstructive lung disease (COPD) and asthma

Sexual dysfunction

Skin diseases such as psoriasis, chronic wounds and inflammation

Vascular diseases such as cerebrovascular disease, aortic aneurysm, thrombosis and peripheral arterial disease

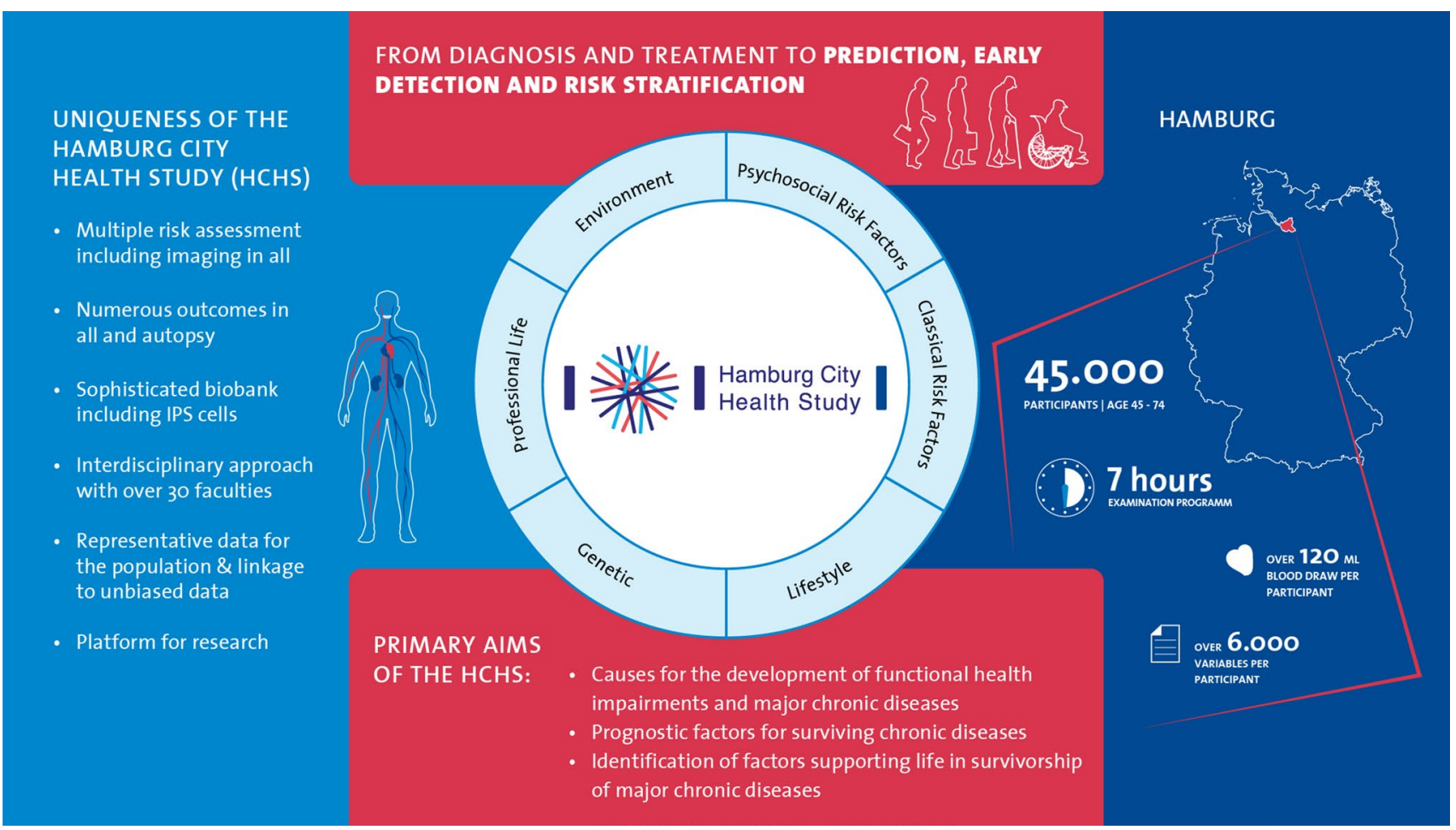

Fig. 1 Summary of aims and uniqueness: the Hamburg City Health Study

\section{Pilot study, timeline and examinations}

From May 08, 2015 until January, 3120161800 volunteers in the age group 18-85 were recruited by a commercial campaign in the leading newspaper from Hamburg and took part to validate the invitation process and train the study nurses in the examination procedures. Moreover, the manageability of the questionnaires was tested. This pilot study led to minor changes in these aspects. The first participant was enrolled on February 08, 2016 in the main 
Box 2 Selected characteristics of the target population: Results from the sample census (microcensus), Hamburg*

\begin{tabular}{|c|c|c|c|c|c|c|c|c|}
\hline \multirow[t]{3}{*}{ Feature } & \multirow[t]{3}{*}{ Category } & \multicolumn{3}{|l|}{ Hamburg } & \multicolumn{3}{|l|}{ Germany } & \multirow[t]{3}{*}{$p$ Value } \\
\hline & & Overall & Men & Women & Overall & Male & Female & \\
\hline & & $\mathrm{N}(\%)$ & $\mathrm{N}(\%)$ & $\mathrm{N}(\%)$ & $\mathrm{N}(\%)$ & $\mathrm{N}(\%)$ & $\mathrm{N}(\%)$ & \\
\hline \multicolumn{9}{|l|}{ Age } \\
\hline & $45-54$ & $274.4(42.9)$ & $139.6(44.3)$ & $134.9(41.5)$ & $13,220(39.8)$ & $6657(40.5)$ & $6563(39.0)$ & \multirow[t]{3}{*}{0.2615} \\
\hline & $55-64$ & $208.5(32.6)$ & $102.4(32.5)$ & $106.1(32.7)$ & $11,698(35.2)$ & $5798(35.3)$ & $5900(35.1)$ & \\
\hline & $65-74$ & $156.9(24.5)$ & $72.9(23.2)$ & $84.0(25.8)$ & 8335 (25.1) & $3966(24.2)$ & $4369(26.0)$ & \\
\hline \multicolumn{9}{|c|}{ Nationality } \\
\hline & German & $1528(84.4)$ & 735 (82.9) & $793(85.8)$ & $73,112(88.5)$ & 35,929 (87.6) & $37,183(89.4)$ & \multirow[t]{2}{*}{$<0.001$} \\
\hline & Other & $282(15.6)$ & $151(17.1)$ & $131(14.2)$ & $9526(11.5)$ & $5103(12.4)$ & 4423 (10.6) & \\
\hline \multicolumn{9}{|c|}{ Marital status } \\
\hline & Married & 679 (37.9) & $339(38.7)$ & $340(37.1)$ & $37,194(45.0)$ & $18,679(45.5)$ & $18,515(44.5)$ & \multirow[t]{4}{*}{$<0.001$} \\
\hline & Single & $881(49.1)$ & 467 (53.2) & $414(45.1)$ & $34,348(41.6)$ & $18,852(45.9)$ & $15,496(37.2)$ & \\
\hline & Divorced & $134(7.5)$ & $53(6.0)$ & $81(8.8)$ & $5717(6.9)$ & $2415(5.9)$ & 3303 (7.9) & \\
\hline & Widowed & $99(5.5)$ & $18(2.0)$ & $82(8.9)$ & $5379(6.5)$ & $1087(2.6)$ & $4292(10.3)$ & \\
\hline \multicolumn{9}{|c|}{ School education $* *$} \\
\hline & Upper secondary education & $721(51.6)$ & $362(53.5)$ & $359(49.9)$ & $22,543(34.6)$ & $11,757(36.7)$ & $10,786(32.6)$ & \multirow[t]{2}{*}{$<0.001$} \\
\hline & Lower secondary education & $675(48.4)$ & $315(46.5)$ & $360(50.1)$ & $42523(65.4)$ & $20271(63.3)$ & $22253(67.4)$ & \\
\hline \multicolumn{9}{|c|}{ Income in $€$} \\
\hline & $500<899$ & $75(9.8)$ & $25(5.5)$ & $50(12.3)$ & $10,256(17.5)$ & $3675(11.9)$ & $6581(23.8)$ & \multirow[t]{9}{*}{$<0.001$} \\
\hline & $900<1299$ & $126(16.4)$ & $49(10.7)$ & $76(18.7)$ & $11984(20.5)$ & $4662(15.1)$ & $7322(26.5)$ & \\
\hline & $1300<1499$ & $79(10.3)$ & $36(7.9)$ & $43(10.6)$ & $5834(10.0)$ & $2807(9.1)$ & $3027(11.0)$ & \\
\hline & $1500<1699$ & $87(11.4)$ & $42(9.2)$ & $45(11.1)$ & $5336(9.1)$ & $2818(9.1)$ & $2518(9.1)$ & \\
\hline & $1700<1999$ & $115(15.0)$ & $59(12.9)$ & $57(14.0)$ & $6538(11.2)$ & $3783(12.2)$ & $2755(10.0)$ & \\
\hline & $2000<2599$ & $69(9.0)$ & $95(20.7)$ & $73(18.0)$ & $8724(14.9)$ & $5654(18.3)$ & $3070(11.1)$ & \\
\hline & $2600<3199$ & $86(11.2)$ & $54(11.8)$ & $32(7.9)$ & $4146(7.1)$ & $2953(9.5)$ & $1193(4.3)$ & \\
\hline & $3200<4499$ & $76(9.9)$ & $55(12.0)$ & $20(4.9)$ & $3611(6.2)$ & $2788(9.0)$ & $822(3.0)$ & \\
\hline & $\geq 4500$ & $53(6.9)$ & $43(9.4)$ & $10(2.5)$ & $2170(3.7)$ & 1827 (5.9) & $344(1.2)$ & \\
\hline
\end{tabular}

*Data retrieved by the Mikrozensus 2016/2017 Statistisches Amt für Hamburg und Schleswig-Holstein. Absolut numbers in 1000 asked persons. The results of a $1 \%$ sample were extrapolated to the current population. The total number is varying due to missing data

**Based on the International Standard Classification of Education (ISCED) which belongs to the UNESCO

study and the last participant is expected to be enrolled on November 30, 2022. The end of the first personal FollowUp is planned 6 years later in 2028.

The participants are contacted by a letter to their home address containing the invitation and an information leaflet providing basic study information. Participants organize their own appointment at the epidemiological study center at the University Medical Center Hamburg-Eppendorf. The appointment is initiated by a study nurse explaining the study rationale and participants are asked to sign informed consent including study participation, an extraction of a skin punch to create induced pluripotent stem cells and either none, one or all of the following options: external, virtual or internal autopsy in the event of death. In the end, participants also sign a consent accepting that both double de-identified and pseudo-anonymized data may be transferred to cooperation partners. Participants are also asked for consent to match their health insurance and pension insurance data with the HCHS dataset. During a 7-h examination participants undergo validated examinations of different organ systems such as anthropometric measures, resting blood pressure measurements, ECG tracings as well as validated physical examinations. Novel parts include detailed cardiovascular, cognitive and oral health phenotyping, skin screening, pulmonary function test, muscle tests and optical coherence tomography (see Box 3 for an overview of all examinations). At the end of the visit, a letter is handed out containing results of all examinations and standardized recommendations to be followed by the participants. Following the discussion with the local ethical committee only clinically relevant results are provided to the participants. Before, during and after the baseline visit validated self-report questionnaires asking for life style and environmental conditions, dietary habits, quality of life, physical and sexual dysfunction, professional life, psychosocial context and burden, digital 
Box 3 Overview of all components of the baseline examination high lightening novel and established clinical practice: the Hamburg City Health Study

\begin{tabular}{|c|c|}
\hline Components of the baseline examination & Variables \\
\hline \multicolumn{2}{|l|}{ Novelty } \\
\hline 2D and 3D transthoracic echocardiography & $\begin{array}{l}\text { Complete screening of aortic-, mitral- and tricuspid-valve. 4D-echocardiography of every partici- } \\
\text { pant of the whole left and right heart. Measurement of ventricular and atrial strain. }\end{array}$ \\
\hline Lung function & Performance of a broad/comprehensive screening by bodyplethysmography. \\
\hline Ophthalmological examination & $\begin{array}{l}\text { Assessment of the objective refraction and subjective visual acuity, imaging of the macular and } \\
\text { papillary retinal layers including intravasal flow visualization using swept source optical coher- } \\
\text { ence tomography. }\end{array}$ \\
\hline Oral examination & $\begin{array}{l}\text { Scaling of periodontitis severity according to the CDC-AAP criteria and whole mouth examination } \\
\text { with assessment of caries (DMFT index) and tooth status. }\end{array}$ \\
\hline Ultrasound of the abdominal aorta & $\begin{array}{l}\text { Evaluation of the infrarenal abdominal aorta by using the b-mode and continuous-wave-Doppler- } \\
\text { mode ultrasound to measure the diameter at the maximum, the outer-to-outer-method is used. } \\
\text { Maximum flow-velocity is measured in the infrarenal aorta. }\end{array}$ \\
\hline Ultrasounds of the peripheral arteries & $\begin{array}{l}\text { B-mode and continuous-wave-Doppler-mode is used to evaluate flow-velocity and plaque burden of } \\
\text { the common femoral, superficial femoral and popliteal arteries. }\end{array}$ \\
\hline \multicolumn{2}{|l|}{ Established clinical practice } \\
\hline 2D and 3D transthoracic echocardiography & $\begin{array}{l}\text { Volumes and function of all four heart chambers by 2D and 3D echocardiography; left ventricular } \\
\text { diastolic function; left ventricular mass. }\end{array}$ \\
\hline Ankle-brachial index (ABI) $[7,8]$ & $\begin{array}{l}\text { Manually measurement (Doppler ultrasound) of systolic blood pressures at the posterior tibial, the } \\
\text { anterior tibial, and the brachial arteries. }\end{array}$ \\
\hline Anthropometry & Assessment of Weight, height and measurement of waist and hip circumferences. \\
\hline Blood pressure measurement & Measurement after 5 min rest and 3 determination sitting and lying. \\
\hline Cognitive function & $\begin{array}{l}\text { Screening for impairment of cognitive function: Mini-Mental State Exam (MMSE), Clock Drawing } \\
\text { Test (CDT); test of cognitive function: verbal fluency, semantic and vocabulary memory, visual } \\
\text { attention and task switching, response inhibition, visuospatial abilities, cognitive processing } \\
\text { speed, executive function. }\end{array}$ \\
\hline Electrocardiography & Assessment with a standard digital 12-lead ECG and 2-min rhythm strip. \\
\hline Lung function & Assessment of spirometry. \\
\hline Muscle status and coordination & Assessment of hand-grip strength, Timed-up and go Test, Chair-Rising Test, Tandem Test. \\
\hline Oral examination & $\begin{array}{l}\text { Assessment of tooth status (DMFT index), pocket probing depths (periodontitis), bleeding on prob- } \\
\text { ing (gingivitis), dental plaque, condition of hard and soft tissues, assessment of craniomandibular } \\
\text { dysfunctions, saliva and sulcus fluid sampling, oral hygiene behavior and oral health literacy. }\end{array}$ \\
\hline Physical activity [9-11] & Objective measure of physical activity with Actigraph. \\
\hline Standardized neurological examination & Assessment of the National Institutes of Health Stroke Scale. \\
\hline Ultrasound of the carotid artery & $\begin{array}{l}\text { B-mode and continuous-wave-Doppler-mode is used to evaluate flow-velocity and plaque burden of } \\
\text { the carotid artery. }\end{array}$ \\
\hline Ultrasounc & The b-mode is used for venous compression ultrasound at the femoral and popliteal vene. \\
\hline
\end{tabular}

media use, medical and family history, occupational history as well as health care utilization are filled out (see Box 4 for an overview of all questionnaires).

Validated risk scores are used to identify individuals at risk for coronary artery disease, atrial fibrillation, heart failure, stroke and dementia. After the baseline investigation, these score-positive participants are invited to the imaging examination including an MRI-examination of the heart and the thoracic aorta and/or brain depending on the target disease [6]. In order to establish a general control group, 1500 random participants are invited to an MRI as well. Participants at risk for osteoporosis or suspected bicuspid aortic valve disease, prostate cancer, HPV-infection or dementia are recommended a further medical clarification.

\section{Laboratory parameters and biobanking}

A panel of basic laboratory analyses are performed on the day of the visit in the study center. The assessed markers include: sodium, potassium, HbA1c, prostate specific antigen (PSA), creatinine, high sensitivity measured CRP, glucose, thyroid stimulating hormone (TSH), triglycerides, total cholesterol, HDL-cholesterol, LDL/ HDL ratio, and N-terminal pro B-type natriuretic peptide (NTproBNP). Furthermore, a complete blood count is performed. Biomaterials used for biobanking include serum, plasma (EDTA, citrate), genomic deoxyribonucleic acid (DNA), ribonucleic acid (RNA) from whole blood and peripheral blood mononuclear cells (PBMCs), blood cells 
Box 4 Overview of all components of the self-reported questionnaires: the Hamburg City Health Study

Component of questionnaire

Overview of dimensions and scales of the baseline and follow-up self-reported questionnaire

Adverse childhood experiences

Alcohol use

Anxiety

Chronotype (single items)

Daytime sleepiness

Depression severity

Dermatology quality of life index

Family history of disease

General practitioners (GP) guided care

Health beliefs

General health perception

Health economics

Informal care

Lifestyle medicine

Living—environment—work

Medical history

Medication-related beliefs

Migraine questionnaire

Occupational exposure to fume, gases, organic or anorganic dust

Optimism, pessimism

Oral health

Patient satisfaction

Quality of life

Resilience

Risk factors and medical history

Sexual dysfunctions

Shift and night work

Social support

Sociodemographic characteristics

Somatic symptom

Utilization of medical services

Significant life events

Smoking behavior

Overview of dimensions and scales of the MRI self-reported questionnaire

Anxiety

Daily life

Depression
Adverse childhood experiences questionnaire (ACE) $[12,13]$

Alcohol use disorders identification test (Audit-C) [14, 15]

Generalized anxiety disorder screener (GAD-7) [16]

Munich chronotype questionnaire (MCTQ) [17, 18]

Epsworth sleepiness skala (ESS) [19]

Patient health questionnaire (PHQ-9 D) [20-22]

Dermatology life quality index (DLQI) [23]

Physical \& psychological diseases of parents

Use of general practitioner

Scale of the German health interview and examination survey for adults (DEGS-39) [24, 25]

Scale of the German health interview and examination survey for adults (DEGS-29) [24, 25]

Costs, EQ-5D-5L, ICER

European Prospective Investigation into Cancer and Nutrition (EPIC): Food Frequency [26] and Physical activity [27]

General housing data, technical data, quality of living, housing and work

Allergies, gynaecology, skin diseases, cardiovascular diseases, cancer, ENT diseases, lung diseases, nephrology, neurology, orthopaedics, other diseases, dental and oral diseases

Beliefs about Medicines Questionnaire (BMQ Short form) [28, 29]

The rostock headache questionnaire (RoKoKo) [30]

Exposure assessment, specifically adapted for HCHS, based on questionnaire of the European community respiratory health survey (ECRHS), occupational module

SWOP

Oral health literacy, oral hygiene, oral hygiene behaviour, oral health care utilization, fluoride prophylaxis, nutrition, dental service utilization [31-33]

Zufriedenheit in der ambulanten Versorgung [Satisfaction in outpatient care] (ZAPA) [34]

European Quality of Life (EQ-5D-5L) [35], [36]

Short-Form Health Survey (SF-8) [37, 38]

RES-6

National cohort study (NAKO) [39]

DSM- 5 criteria

career history, jobs with shift and night work [40, 41]

F-SozU-K14

i.e. Gender, age, nationality, mother language, religion

Patient Health Questionnaire (PHQ-15) PHQ-15 [42, 43]

Scales of the German Health Interview and Examination Survey for Adults (DEGS-9, DEGS-10, ÖKO-DEGS-15) [24]

Social reactivity reaction scale (SRRS)

Fagerström questionnaire, smoking anamnesis

Generalized anxiety disorder (GAD-2) [44]

Social interaction, cognitive deficits, own psychological diseases $\&$ in the family

Patient health questionnaire (PHQ-9) [20, 21] 
Box 4 (continued)

Component of questionnaire

Emotional regulation

Emotional well-being

Health advice

Health behaviour measures

Health care satisfaction

Health literacy

Health risks perception

Media consumption

Medication-related beliefs

Personality

Quality of life

Self-efficacy

Significant life events

Somatic Symptoms
Questions from the response styles questionnaire (RSQ-D) [45, 46]

Current personal condition

Scale of the German health interview and examination survey for adults (DEGS-11) [24]

Scale of the German health interview and examination survey for adults (DEGS-20) [24]

Zufriedenheit in der ambulanten Versorgung/[Satisfaction in outpatient care] (ZAPA) [34, 46]

Patient Activation Measure (PAM-13)

Patterns of media consumption

Beliefs about medicines questionnaire (BMQ Short form) [28, 29]

Eysenck personality questionnaire (EPG-RK) [47]

Short-Form Health Survey (SF-8) [37, 38]

Skala zur Allgemeinen Selbstwirksamkeits-erwartung [Self-efficacy scale] (SWE)

Social readjustment rating scale (SRRS)

Somatic symptom disorder-B criteria scale SSD-12) [48]

Somatic Symptom Scale (SSS-8) [43] (erythrocytes, PBMCs), urine, saliva as well as tooth fluid and tonsils swabs. Additionally, from a random subset of study participants, skin stanza are collected of which fibroblasts are separated. These fibroblasts will be used for the generation of human induced pluripotent stem cells (hiPSCs) (for an overview of all biomaterials collected and planned measurements see Box 5). Subsequently, the biomaterial will be examined by state-of-the-art and innovative, high throughput approaches including analyses on the different OMICS levels such as genomics, transcriptomics, proteomics and lipidomics profiling. One part of the biobank is used for research projects within the first
6 years, the second part is stored for projects performed during the studies follow up and in the future.

\section{Follow-up}

Following the date of baseline examination, all participants will be contacted by mail containing a questionnaire which specifically ask participants to report any major medical event, medication, nutrition and lifestyle changes, physical and mental health, sexual dysfunction and overall quality of life as well as health care use. Participants are also asked to provide discharge letters or any kind of further information

Box 5 Overview of all collected biospecimen and purposes: the Hamburg City Health Study

\begin{tabular}{|c|c|}
\hline Biospecimen & Novelty \\
\hline Serum & $\begin{array}{l}\text { Measurement of routine biomarkers as well as innovative markers such as non-coding RNA, proteomics, metabolomics and } \\
\text { viruses }\end{array}$ \\
\hline EDTA plasma & Measurement of routine biomarkers as well as innovative markers such as ncRNA, proteomics, metabolomics and viruses \\
\hline Citrat plasma & Measurement of routine biomarkers and innovative markers of coagulation cascade \\
\hline Genomic DNA & Measurement genomic and epigenomic markers \\
\hline Whole blood RNA & Measurement of gene expression and expression of non-coding RNA \\
\hline Erythrocytes & Measurement of innovative biomarkers and protein activity \\
\hline PBMC & Generation of iPS cells, and cell culture experiments, Gene expression and measurement of circulating tumorcells (CTC) \\
\hline Urine & Measurement of innovative markers \\
\hline Saliva & Measurement of innovative biomarkers and oral microbiome \\
\hline Tooth fluid & Measurement of oral microbiome \\
\hline Tonsils swab & Measurement of oral microbiome and viruses \\
\hline Skin Tissue & Generation of iPS cells \\
\hline
\end{tabular}


on their health such as diagnostic findings or images. This contact takes place on an annual basis for 5 years. An endpoint-committee will review all collected information for special endpoints. After 6 years all participants are invited to undergo the same examination and procedures as in the baseline visit. On a continuous basis the study center is in contact with public authorities and the cancer register about vital status, cause of death and cancer incidence and in contact with involved health and pension insurances to match with related individual routine data.

\section{Statistical analysis plan}

The integrity of the collected data in the databases are controlled by detailed, predefined quality control algorithms according to standardized operation procedures (SOP) concerning detection of outliers, logically implausibility, or detect mistaken identity. Only quality controlled data will be used for statistical analyses.

In the analyses of baseline data methods for cross-sectional analyses will be applied. Univariate statistics for categorical variables will be presented as counts and proportions, and numeric variables will be presented as means, percentiles and standard deviations. Associations between baseline characteristics will be estimated by means generalized linear regression models.

For the full cohort as well as for sub-cohorts, time-toevent methods constitute the major approach for identifying and assessing risk factors for mortality and incidence or progression of diseases. Thus, when studying rates of a single event type, e.g., overall mortality, regression models for censored data will be used to simultaneously investigate the effects of risk factors of interest while adjusting for potential confounders.

In all analyses, effect estimates will be presented both, in relative terms as prevalence or risk ratios, and in absolute terms as means, rates or risks, the latter to assess public health implications. All effect estimates and statistical summaries will be presented with $95 \%$ confidence intervals, and, when appropriate, adjusted for multiple testing. For diseasespecific mortality or endpoints other than death, competing risks will be accounted for. For repeated measurements in the second examination, regression models for longitudinal data will be employed. In all analyses observations may be correlated either in time (repeated measures) or between subjects (e.g. relatives). Appropriate statistical methods will be used to account for correlated observations, e.g. by means of mixed effect models, or in marginal models by means of generalized estimating equations (GEE). Special attention will be paid to potentially informative drop-out or failure to obtain further measurements due to the occurrence of a specific event. These issues will be addressed by joint models for longitudinal and time-to-event data. In cases of a substantial amount of missingness in dependent or independent variables, multiple imputation will be incorporated in the analysis, as a supplement to the complete case analysis. To ensure transparency and reproducibility of results, all statistical methods and codes will be described and stored centrally as a supplement to study protocols. This material will be available to other researchers upon request.

\section{Ethical concerns}

During the study conception, the local ethics committee of the Landesärztekammer Hamburg (State of Hamburg Chamber of Medical Practitioners, PV5131) was consulted and its approval for the study protocol as well as the approval by the Data Protection Commissioner of the University Medical Center of the University Hamburg-Eppendorf and the Data Protection Commissioner of the Free and Hanseatic City of Hamburg were obtained. The study has been registered at ClinicalTrial.gov (NCT03934957). The procedures set out in this study, pertaining to the conduct, evaluation, and documentation, are designed to ensure that all persons involved in the study abide by Good Clinical Practice (GCP), Good Epidemiological Practice (GEP) and the ethical principles described in the current revision of the Declaration of Helsinki. The study will be carried out in keeping with local legal and regulatory requirements. The requirements of the GCP and GEP regulation will be adhered to. In order to be admitted to HCHS, all participants are to consent to participate only after the nature and scope of the study have been explained to and understood by them. Written informed consent is obtained from all participants. The examinations were chosen because of non-invasive nature of acquisition and standardized testing to assess intermediate phenotypes of the different diseases. Well-characterized intermediate phenotypes are of great value for screening, patient monitoring and in the context with clinical trials. At the end of the baseline- and MRI- examinations, all participants receive a standardized report of all relevant clinical results with a possible recommendation of referral to the general practitioner. Recommendations for all findings were defined before study start and standardized. Findings, which need clinical referral immediately, are defined as well and reported directly to the participants. If required, participants are also accompanied to the emergency department of the University Medical Center Hamburg-Eppendorf by a member of the study staff.

\section{Data protection concerns}

During the study conception, the Data Protection Commissioner of the University Medical Center Hamburg-Eppendorf and the Data Protection Commissioner of the Free 
and Hanseatic City of Hamburg were consulted and their approval for the study protocol was obtained (D4/17.06-22). The requirements of the Federal Data Protection Law (Bundesdatenschutzgesetz), the European General Data Protection Regulation and the law of Hamburg Hospitals (Hamburger Krankenhausgesetz) will be adhered to. Subjects will be identified solely by means of an individual identification code and data are only collected, stored and analysed pseudo-anonymized. To enhance data confidentiality, data, lab samples and genetic results have different identification numbers. The identification numbers will change between data collection, storage and disclosure. To avoid mistakes in assigning the right identification number, the pseudoanonymization is conducted electronically. All data will be stored at the study centre and all analytical activity will take place at dedicated workstations situated at the study centre. Extensive surveillance and login of all activity will be carried out.

\section{Strengths and limitations}

Most importantly, the HCHS is a prospective populationbased cohort study, which enables cause and effect analyses investigating major risk factors for a number of symptoms and chronic diseases. In addition, the extensive annual follow-up also contributes to the advantages of the HCHS. Further, the high number of examinations including a range of novel approaches in different disciplines contributes to the fact that data from the HCHS may unravel hitherto unknown causal associations. In line with this consideration, the extensive analyses planned with the use of biological samples giving basic genomic information in combination with phenotypic data will provide a rich data source for advanced analyses. The high number of participants secures that even relatively rare outcomes may be investigated. Furthermore, it is the ambition to link the data from the HCHS to socioeconomic information and individual records indicating information about diseases, prescribed medications, in- and outpatient health care use as well as sick leave etc. The cohort recruitment allows that prevalent cases of most chronic diseases will be enrolled and thereby opens up for early cross-sectional analyses investigating associations leading to hypothesis driven studies when the first 10.000 participants are included and when the cohort is complete. In line with this consideration, one may also point to the possibility of describing the distribution of diseases associated with patterns of several factors, i.e. socioeconomic status in diabetes patients, nutrition and lifestyle factors in dementia or the association between skin disorder and dental status. The design also offers an opportunity to conduct more complicated disease trajectory analyses using patterns of symptoms and diseases as both causes and effects. It is also of vital importance that this study offers an opportunity to investigate determinants of survival and survivorship in accordance with the aims of the study.

Even prospective cohort studies will have limitations due to selection bias in participation and recall bias. This study mostly relies on the recall of participants as the method of achieving information about central outcomes during the 6 years of follow-up. This limitation will be addressed by obtaining data from health insurance and pension funds. Compared to cohort studies investigating risk factors of diseases characterized by high incidence rates the waiting time for incident outcomes in diseases having low incidence rates is longer. Conducting a multidimensional study also reduces the number of participants with complete data, and as funding is limited, this problem represents a true limitation of the data set, which in the end will be less than a $100 \%$ complete. From a more technical point of view, some data may not be collected due to participants wish, lack of staff or technical malfunction of equipment. To address this limitation, standardized quality controls are performed weekly to detect missing data in order to ensure that no systematic processes are in function. Birthday, Christmas cards and annual newsletters will remind the participant to inform the study centre in the event of a medical endpoint. A number of standardized operating procedures for the recruitment, collecting and storage of data, quality control and analyses will be established. To avoid endpoint misclassification, an interdisciplinary endpoint committee will render an expert opinion on the basis of discharge letters or further information. The integrity of the collected data in the databases is controlled by detailed, predefined quality control algorithms according to standardized operating procedures in batches concerning detection of outliers, logically implausibility, or detect mistaken identity. Only quality-controlled data are used for statistical analyses.

\section{Conclusion}

In future, data from HCHS will strongly contribute to our knowledge about risk factors for and prognostic factors in major chronic diseases, survival and survivorship. It will be a unique source due to the combination of self-reported data, detailed imaging data, a vast number of biological information and unbiased administrative data established independent of the hypothesis of the study. It is the aspiration that the inclusion of novel aspects in all exposure assessment methods concurrently with well-established, traditional epidemiological tools across all exposures and outcome will help in achieving information of such quality so that it can feed directly into public health policy with regard to prevention and survivorship related aspects. In line with this aspiration, 
the use of the data in real-life clinic is also one of the main intention in this study.

Acknowledgements The first author named is lead and corresponding author. The second, third and last authors are responsible for the supervision. All other authors are listed in alphabetical order. We describe contributions to the paper using the CRediT contributor role taxonomy. Writing-Original Draft: Jagodzinski; Writing-Review \& Editing: Jagodzinski, Johansen, Blankenberg; Conceptualization: Blankenberg, Koch-Gromus, Gerloff, Adam, Jagodzinski, Zeller; Funding Acquisition: all, Investigation: all. The publication has been approved by the Steering Board of the Hamburg City Health Study. The authors acknowledges the participants of the Hamburg City Health Study and cooperation partners, patrons and the Deanery from the University Medical Centre Hamburg-Eppendorf for supporting the Hamburg City Health Study. Special thanks applies to the staff at the Epidemiological Study Centre for conducting the study. The participating institutes and departments from the University Medical Center Hamburg-Eppendorf contribute with individual and scaled budgets to the overall funding. The study is also supported by the Innovative medicine initiative (IMI) under Grant No. 116074, by the Fondation Leducq under Grant Number 16 CVD 03, by the euCanSHare Grant Agreement No. 825903-euCanSHare H2020 and the DFG under project Grant TH1106/5-1; AA93/2-1. The licence for the Food Frequency and Physical activity is provided by the DIFE. Technical equipment is provided by SIEMENS according to a contract for 12 years as well as by the Schiller AG on a loan basis for 6 years and by Topcon on a loan basis from 2017 until 2022. The Hamburg City Health Study is additionally supported by an unrestricted Grant (2017-2022) by Bayer. Project related analyses are supported by Amgen, Astra Zeneca, BASF, Deutsche Gesetzliche Unfallversicherung (DGUV), DKFZ, DZHK, Novartis, Seefried Stiftung and Unilever. The study is further supported by donations from the "Förderverein zur Förderung der HCHS e.V.", TEPE (2014) and Boston Scientific (2016). A current list of the supporter is online available on www.uke.de/hchs.

\section{Compliance with ethical standards}

Conflict of interests Ghazal Aarabi: Has received project related funding by the DFG. Gerhard Adam: Nothing to disclose. Sven Anders: received research funding from German Ministry of Science and Education (BMBF) and Rickertsen Foundation. Matthias Augustin: Nothing to disclose. Ramona Bei der Kellen: Nothing to disclose. Thomas Beikler: Nothing to disclose. Christian-Alexander Behrendt: has received research funding from the German Federal Joint Committee (Grant No. 01VSF16008 and 01VSF18035). Christian S. Betz: Nothing to disclose relating to HCHS. Carsten Bokemeyer: Nothing to disclose. Katrin Borof: Nothing to disclose. Peer Briken: Nothing to disclose. Chia-Jung Busch: Has received project related funding by the DKFZ. Christian Büchel: Nothing to disclose. Stefanie Brassen: Nothing to disclose. Sebastian Debus received institutional Grants from COOK, Vascutek and Bayer. He has also received honoraria as member of Bayer Advisory boards. Larissa Eggers: Nothing to disclose. Jens Fiehler: received research funding from German Ministry of Science and Education (BMBF), German Ministry of Economy and Innovation (BMWi), German Research Foundation (DFG), European Union (EU), Hamburgische Investitions- und Förderbank (IFB), Medtronic, Microvention, Philips, Stryker; received honoraria as consultant or speaker for Acandis, Boehringer Ingelheim, Cerenovus, Covidien, Evasc Neurovascular, MD Clinicals, Medtronic, Medina, Microvention, Penumbra, Route92, Stryker, Transverse Medical. Jürgen Gallinat: has received research funding from the German Federal Ministry of Education and Research, German Science Foundation, and speaker fees from Lundbeck, Janssen-Cilag, Lilly and Otsuka. Christian Gerloff: received funding from German Research Council (DFG), European Union, Federal Ministery of Education and Research (BMBF), German Statutory Pension Insurance Scheme (RV Nord), National Innovation Fond, Wegener Foundation, and Schilling Foundation; received honoraria as speaker or consultant from Abbott, Amgen, Bayer Vital, Bristol-Myers-Squibb, Boehringer Ingelheim, Sanofi Aventis, and Prediction Biosciences. Evaldas Girdauskas: Nothing to disclose. Martin Gosau: Nothing to disclose. Uwe Koch- Gromus: Nothing to disclose. Markus Graefen: Nothing to disclose. Martin Härter: received funding from the Federal Ministery of Education and Research (BMBF) for the project PERGOLA. Volker Harth: received funding from European Union, Federal Ministery of Education and Research (BMBF), Federal Ministery of Health (BMG), and Deutsche Gesetzliche Unfallversicherung (DGUV). Christoph Heidemann: Nothing to disclose. Guido Heydecke: Nothing to disclose. Tobias Huber: Nothing to disclose relating to HCHS. Yassin Hussein: Nothing to disclose. Annika Jagodzinski: Has received project related funding by Amgen and an unrestricted Grant by Bayer. Christoffer Johansen: Nothing to disclose. Marvin O. Kampf: Nothing to disclose. Olaf von dem Knesebeck: Nothing to disclose. Alexander Konnopka: Nothing to disclose. Hans-Helmut König: Nothing to disclose. Robert Kromer: received funding from the Federal Ministry of Economics and Technology, European Union, Santen and StullnPharma; received honoraria as speaker from Bayer, Novartis, Santen and Topcon. Christian Kubisch: Nothing to disclose. Simone Kühn: has been funded by the German Science Foundation, the European Union (ERC-2016-StG-Self-Control-677804) and a Fellowship from the Jacobs Foundation. Sonja Loges: Nothing to disclose with regards to this publication. Bernd Löwe: Nothing to disclose. Gunnar Lund: Nothing to disclose. Christian Meyer: is supported by the German Center for Cardiovascular Research (DZHK, 81Z0710112). Lina Nagel: Nothing to disclose Albert Nienhaus: Received funds from the German Ministry of Health (BMG), the European Union Social Dialog Fund, Institution for Statutory Accident Insurance and Prevention in the Health and Welfare Services, and ZEIT-Stiftung. Klaus Pantel: Nothing to disclose. Elina Petersen: Nothing to disclose. Klaus Püschel: Nothing to disclose. Hermann Reichenspurner: received honoraria as consultant for Abiomed und Medtronic. Guido Sauter: Nothing to disclose. Martin Scherer: Nothing to disclose. Katharina Scherschel: is supported by the German Center for Cardiovascular Research (DZHK, 81Z0710112). Ulrich Schiffner: has received honoraria for lectures from 3 M, CP Gaba, and DMG. Renate Schnabel: has received funding from the European Research Council (ERC) under the European Union's Horizon 2020 research and innovation programme (Grant Agreement No 648131), German Ministry of Research and Education (BMBF 01ZX1408A) and German Center for Cardiovascular Research (DZHK e.V.) (81Z1710103). Holger Schulz: Nothing to disclose. Ralf Smeets: Nothing to disclose. Vladislavs Sokalskis: Nothing to disclose. Martin S. Spitzer has received research funding from Novartis, Clanotech, Croma Pharma Topcon and Flouron. He received honoraria for lectures from Novartis, Bayer, Alcon, Allergan, Alimera Sciences and for consulting from Bayer, SHS and Altacor. Claudia Terschüren: received funding from Deutsche Gesetzliche Unfallversicherung (DGUV). Imke Thederan: Nothing to disclose. Götz Thomalla: received funding from the German Research Council (DFG), European Union's Horizon 2020 research and innovation programme, German Federal Ministry for Economic Affairs and Energy (BMWi), Germany's Federal Joint Committee (G-BA) Innovation Fund (Innovationsfonds), and has received honoraria as speaker or consultant from Acandis, Bayer Vital, Bristol-Myers-Squibb/Pfizer, Boehringer Ingelheim, Daiichi Sankyo and Stryker. Benjamin Waschki: Nothing to disclose. Karl Wegscheider: Nothing to disclose. Jan-Per Wenzel: Nothing to disclose. Susanne Wiese: Nothing to disclose. Birgit-Christiane Zyriax: has received project related funding by BASF, Unilever and DIFE. Tanja Zeller: is supported by the German Center for Cardiovascular Research (DZHK, 81Z0710102). This project has received funding from the European Union's Horizon 2020 research and inno- 
vation programme under Grant Agreement No. 825903, the Innovative medicine initiative (IMI) under Grant No. 116074 and the Fondation Leducq under Grant Number 16 CVD 03. Stefan Blankenberg: is supported by the Innovative medicine initiative (IMI) under Grant No. 116074, the Fondation Leducq under Grant Number 16 CVD 03, Siemens, Bayer, Astra Zeneca, Deutsche Gesetzliche Unfallversicherung (DGUV) and Novartis for project related analyses.

Open Access This article is distributed under the terms of the Creative Commons Attribution 4.0 International License (http://creativeco mmons.org/licenses/by/4.0/), which permits unrestricted use, distribution, and reproduction in any medium, provided you give appropriate credit to the original author(s) and the source, provide a link to the Creative Commons license, and indicate if changes were made.

\section{References}

1. Lopez $\mathrm{AD}$, et al. Remembering the forgotten non-communicable diseases. BMC Med. 2014;12:200.

2. What is the impact of noncommunicable diseases on national health expenditures: a synthesis of available data. Financing WDfHS, 2011.

3. Health at a Glance: Europe 2016. Health Statistics, 2016.

4. Wong ND, Levy D. Legacy of the framingham heart study: rationale, design, initial findings, and implications. Glob Heart. 2013;8(1):3-9.

5. Schleswig-Holstein, S.A.f.H.u., STATISTISCHE BERICHTE: Bevölkerung in Hamburg-Auszählung aus dem Melderegister. 2018.

6. Bohnen S, et al. Cardiovascular magnetic resonance imaging in the prospective, population-based, Hamburg City health cohort study: objectives and design. J Cardiovasc Magn Reson. 2018;20(1):68.

7. Greenland P, Smith SC Jr, Grundy SM. Improving coronary heart disease risk assessment in asymptomatic people. Circulation. 2001;104(15):1863-7.

8. Collaboration ABI. Ankle brachial index combined with Framingham risk score to predict cardiovascular events and mortality: a meta-analysis. JAMA J Am Med Assoc. 2008;300(2):197.

9. Massy-Westropp NM, et al. Hand grip strength: age and gender stratified normative data in a population-based study. BMC Res Notes. 2011;4(1):127.

10. Martin JL, Hakim AD. Wrist actigraphy. Chest J. 2011;139(6):1514-27.

11. Marino M, et al. Measuring sleep: accuracy, sensitivity, and specificity of wrist actigraphy compared to polysomnography. Sleep. 2013;36(11):1747.

12. Wingenfeld $\mathrm{K}$, et al. The reliable, valid and economic assessment of early traumatization: first psychometric characteristics of the German version of the adverse childhood experiences questionnaire (ACE). Psychother Psychosom Med Psychol. 2011;61(1):e10-4.

13. Wingenfeld K, Schäfer I, Terfehr K, Grabski H, Grabe HJ, Driessen M, Löwe B, Spitzer C. Reliable, valide und ökonomische Erfassung früher Traumatisierung: Erste psychometrische charakterisierung der deutschen Version des adverse childhood experiences questionnaire (ACE). Psychother Psychosom Med Psychol. 2011;61:42-5.

14. Bush K, et al. The AUDIT alcohol consumption questions (AUDIT-C): an effective brief screening test for problem drinking. Ambulatory care quality improvement project (ACQUIP). Alcohol use disorders identification test. Arch Int Med. 1998;158(16):1789-95.
15. Rumpf HJ, Meyer C, Hapke U, John U, Deutsche version des alcohol use disorders identification test (AUDIT). Elektronisches Handbuch zu Erhebungsinstrumenten im Suchtbereich (EHES). 2001.

16. Lowe B, et al. Validation and standardization of the generalized anxiety disorder screener (GAD-7) in the general population. Med Care. 2008;46(3):266-74.

17. Roenneberg T, Wirz-Justice A, Merrow M. Life between clocks: daily temporal patterns of human chronotypes. J Biol Rhythms. 2003;18(1):80-90.

18. Zavada A, et al. Comparison of the Munich chronotype questionnaire with the Horne-Ostberg's morningness-eveningness score. Chronobiol Int. 2005;22(2):267-78.

19. Bloch KE, et al. German version of the epworth sleepiness scale. Respiration. 1999;66(5):440-7.

20. Lowe B, et al. Diagnosing ICD-10 depressive episodes: superior criterion validity of the patient health questionnaire. Psychother Psychosom. 2004;73(6):386-90.

21. Lowe B, et al. Monitoring depression treatment outcomes with the patient health questionnaire-9. Med Care. 2004;42(12):1194-201.

22. Kroenke K, Spitzer RL, Williams JB. The PHQ-9: validity of a brief depression severity measure. J Gen Int Med. 2001;16(9):606-13.

23. Augustin M, et al. Quality of life in skin diseases: methodological and practical comparison of different quality of life questionnaires in psoriasis and atopic dermatitis. Hautarzt. 1999;50(10):715-22.

24. Gosswald A, et al. DEGS: german health interview and examination survey for adults. A nationwide cross-sectional and longitudinal study within the framework of health monitoring conducted by the Robert Koch Institute. Bundesgesundheitsblatt Gesundheitsforschung Gesundheitsschutz. 2012;55(6-7):775-80.

25. Gosswald A, Lange M, Kamtsiuris P, Kurth BM. DEGS: studie zur gesundheit erwachsener in Deutschland. BundesgesundheitsblattGesundheitsforschung-Gesundheitsschutz. 2012;55(6-7):775-80.

26. Nothlings $U$, et al. Fitting portion sizes in a self-administered food frequency questionnaire. J Nutr. 2007;137(12):2781-6.

27. Wareham NJ, et al. Validity and repeatability of the EPICNorfolk physical activity questionnaire. Int $\mathrm{J}$ Epidemiol. 2002;31(1):168-74

28. Opitz U, Glattacker M, Bengel J, Jäckel WH, Horne R. Der" beliefs about medicines questionnaire" -übersetzung und erste methodische prüfung an patienten mit fibromyalgie. DRV-Schriften. 2008;77:99-100.

29. Horne R, Weinman J, Hankins M. The beliefs about medicines questionnaire: the development and evaluation of a new method for assessing the cognitive representation of medication. Psychol Health. 1999; 14:1-24.

30. Muller B, et al. The rostock headache questionnaire ("Rokoko") validation of a tool to screen and to qualify primary headaches. Fortschr Neurol Psychiatr. 2014;82(3):145-8.

31. Jordan RA, et al. The fifth german oral health study (funfte deutsche mundgesundheitsstudie, DMS V)—rationale, design, and methods. BMC Oral Health. 2014;14:161.

32. Sabbah W, et al. Social gradients in oral and general health. J Dent Res. 2007;86(10):992-6.

33. Naghibi Sistani MM, et al. New oral health literacy instrument for public health: development and pilot testing. J Investig Clin Dent. 2014;5(4):313-21.

34. Scholl I, Hölzel L, Härter M, Dierks ML, Bitzer EM, Kriston L. Fragebogen zur zufriedenheit in der ambulanten versorgungschwerpunkt patientenbeteiligung (ZAPA). Klin Diagn Eval. 2011;4(1):50-62.

35. EuroQol Group. EuroQol-a new facility for the measurement of health-related quality of life. Health Policy. 1990;16(3):199-208. 
36. Hinz A, et al. The quality of life questionnaire EQ-5D-5L: psychometric properties and normative values for the general German population. Qual Life Res. 2014;23(2):443-7.

37. Beierlein V, Morfeld M, Bergelt C, Bullinger M, Brähler E. Messung der gesundheitsbezogenen Lebensqualität mit dem SF-8 - Deutsche Normdaten aus einer repräsentativen schriftlichen Befragung. Diagnostica. 2012;58(3):145-53.

38. Ware JE, Kosinski M, Dewey JE, Gandek B, How to score and interpret single-item health status measures: a manual for users of the SF-8 health survey. Quality Metric Incorpotated. 2001b.

39. The German National Cohort. The German national cohort aims, study design and organization. Eur J Epidemiol. 2014;29(5):371-82.

40. Stevens RG, et al. Considerations of circadian impact for defining 'shift work' in cancer studies: iARC working group report. Occup Environ Med. 2011;68(2):154-62.

41. Rabstein $\mathrm{S}$, et al. Night work and breast cancer estrogen receptor status-results from the German GENICA study. Scand J Work Environ Health. 2013;39(5):448-55.

42. Kroenke K, Spitzer RL, Williams JB. The PHQ-15: validity of a new measure for evaluating the severity of somatic symptoms. Psychosom Med. 2002;64(2):258-66.

43. Gierk B, et al. Assessing somatic symptom burden: a psychometric comparison of the patient health questionnaire-15 (PHQ$15)$ and the somatic symptom scale-8 (SSS-8). J Psychosom Res. 2015;78(4):352-5.
44. Wild B, et al. Assessing generalized anxiety disorder in elderly people using the GAD-7 and GAD-2 scales: results of a validation study. Am J Geriatr Psychiatry. 2014;22(10):1029-38.

45. Huffziger S, Kuhner C. Die ruminationsfacetten brooding und reflection: eine psychometrische evaluation der deutschsprachigen version RSQ-10D. Z Fur Klin Psychol Psychother. 2012;41:38-46.

46. Nolen-Hoeksema S. Responses to depression and their effects on the duration of depressive episodes. J Abnorm Psychol. 1991;100(4):569-82.

47. Ruch W. Die revidierte fassung des eysenck personality questionnaire und die konstruktion des Deutschen EPQ-R bzw. Zeitschrift für Differentielle und Diagnostische Psychologie: EPQ-RK; 1999.

48. Toussaint A et al., Detecting DSM-5 somatic symptom disorder: criterion validity of the patient health questionnaire-15 (PHQ-15) and the somatic symptom scale-8 (SSS-8) in combination with the somatic symptom disorder-B Criteria Scale (SSD-12). Psychol Med. 2019;1-10.

Publisher's Note Springer Nature remains neutral with regard to jurisdictional claims in published maps and institutional affiliations.

\section{Affiliations}

Annika Jagodzinski ${ }^{1,2,3}$ (1) Christoffer Johansen ${ }^{3,4,5,6} \cdot$ Uwe Koch-Gromus $^{7} \cdot$ Ghazal Aarabi $^{8} \cdot$ Gerhard Adam $^{9}$. Sven Anders ${ }^{10}$. Matthias Augustin ${ }^{11}$. Ramona B. der Kellen ${ }^{3}$. Thomas Beikler ${ }^{12}$. Christian-Alexander Behrendt ${ }^{13}$. Christian S. Betz ${ }^{14}$. Carsten Bokemeyer ${ }^{15}$. Katrin Borof ${ }^{3}$. Peer Briken ${ }^{16}$. Chia-Jung Busch ${ }^{14}$. Christian Büchel ${ }^{17}$. Stefanie Brassen ${ }^{17}$. Eike S. Debus ${ }^{13}$. Larissa Eggers ${ }^{3}$. Jens Fiehler ${ }^{18}$. Jürgen Gallinat ${ }^{19}$. Simone Gellißen ${ }^{18}$. Christian Gerloff $^{20}$. Evaldas Girdauskas ${ }^{21}$ - Martin Gosau ${ }^{22}$. Markus Graefen ${ }^{23}$ - Martin Härter ${ }^{24}$. Volker Harth ${ }^{25}$. Christoph Heidemann ${ }^{3}$. Guido Heydecke ${ }^{8}$. Tobias B. Huber ${ }^{26}$. Yassin Hussein ${ }^{3}$. Marvin O. Kampf ${ }^{3}$. Olaf von dem Knesebeck ${ }^{27}$. Alexander Konnopka ${ }^{28} \cdot$ Hans-Helmut König ${ }^{28} \cdot$ Robert Kromer $^{29}$. Christian Kubisch $^{30}$. Simone Kühn ${ }^{19}$. Sonja Loges ${ }^{15,34}$. Bernd Löwe ${ }^{31}$. Gunnar Lund ${ }^{9}$. Christian Meyer ${ }^{2,32}$. Lina Nagel ${ }^{3}$ - Albert Nienhaus ${ }^{33} \cdot$ Klaus Pantel $^{34}$ - Elina Petersen ${ }^{3} \cdot$ Klaus Püschel $^{10} \cdot$ Hermann Reichenspurner $^{21}$. Guido Sauter ${ }^{35} \cdot$ Martin Scherer $^{36} \cdot$ Katharina Scherschel $^{2,32} \cdot$ Ulrich Schiffner $^{12} \cdot$ Renate B. Schnabel $^{1,2}$. Holger Schulz ${ }^{24}$. Ralf Smeets ${ }^{22}$. Vladislavs Sokalskis ${ }^{3}$ Martin S. Spitzer ${ }^{29}$. Claudia Terschüren ${ }^{25} \cdot$ Imke Thederan $^{23}$. Tom Thoma ${ }^{3} \cdot$ Götz Thomalla $^{20} \cdot$ Benjamin Waschki $^{1,2,38} \cdot$ Karl Wegscheider $^{6} \cdot$ Jan-Per Wenzel ${ }^{1,3} \cdot$ Susanne Wiese $^{3}$. Birgit-Christiane Zyriax ${ }^{2,37} \cdot$ Tanja Zeller $^{1,2,3} \cdot$ Stefan Blankenberg ${ }^{1,2}$

Annika Jagodzinski

a.jagodzinski@uke.de

1 Department of General and Interventional Cardiology, University Heart and Vascular Center, Hamburg, Germany

2 German Center for Cardiovascular Research (DZHK) Partner Site Hamburg/Lübeck/Kiel, Munich, Germany

3 Epidemiological Study Center, University Medical Center Hamburg-Eppendorf (UKE), Hamburg, Germany

4 Oncology Clinic, Finsen Center, Copenhagen, Denmark

5 Survivorship Research Unit, The Danish Cancer Society Research Center, Copenhagen, Denmark

6 Institute for Medical Biometry and Epidemiology (IMBE), University Medical Center Hamburg-Eppendorf (UKE), Hamburg, Germany
7 Faculty of Medicine, University Medical Center Hamburg-Eppendorf (UKE), Hamburg, Germany

8 Department of Prosthetic Dentistry, Center for Dental and Oral Medicine, University Medical Center Hamburg-Eppendorf, Hamburg, Germany

9 Department of Diagnostics and Interventional Radiology and Nuclear Medicine, University Medical Center Hamburg-Eppendorf (UKE), Hamburg, Germany

10 Department for Forensic Medicine, University Medical Center Hamburg-Eppendorf (UKE), Hamburg, Germany

11 Institute for Health Services Research in Dermatology, University Medical Center Hamburg-Eppendorf (UKE), Hamburg, Germany

12 Department of Periodontics, Preventive and Restorative Dentistry, University Medical Center Hamburg-Eppendorf (UKE), Hamburg, Germany 
13 Department of Vascular Medicine, University Heart and Vascular Center, Hamburg, Germany

14 Department of Otolaryngology, Head and Neck Surgery, Head and Neurocenter, University Medical Center Hamburg-Eppendorf (UKE), Hamburg, Germany

15 Department of Oncology, Hematology, BMT with Section Pneumology, University Medical Center Hamburg-Eppendorf (UKE), Hamburg, Germany

16 Institute for Sexual Research and Forensic Psychiatry, University Medical Center Hamburg-Eppendorf (UKE), Hamburg, Germany

17 Institute for Systemic Neurosciences, University Medical Center Hamburg-Eppendorf (UKE), Hamburg, Germany

18 Clinic of Neuroradiological Diagnostics and Intervention, University Medical Center Hamburg-Eppendorf (UKE), Hamburg, Germany

19 Department of Psychiatry and Psychotherapy, University Medical Center Hamburg-Eppendorf (UKE), Hamburg, Germany

20 Department of Neurology, University Medical Center Hamburg-Eppendorf (UKE), Hamburg, Germany

21 Department for Cardiovascular Surgery, University Heart and Vascular Center, Hamburg, Germany

22 Department of Oral and Maxillofacial Surgery, University Medical Center Hamburg-Eppendorf (UKE), Hamburg, Germany

23 Prostate Cancer Center, Martini-Clinic, University Medical Center Hamburg-Eppendorf (UKE), Hamburg, Germany

24 Department of Medical Psychology, University Medical Center Hamburg-Eppendorf (UKE), Hamburg, Germany

25 Institute for Occupational and Maritime Medicine (ZfAM), University Medical Center Hamburg-Eppendorf (UKE), Hamburg, Germany

26 Medical Clinic and Polyclinic III, University Medical Center Hamburg-Eppendorf (UKE), Hamburg, Germany
27 Institute for Medical Sociology, University Medical Center Hamburg-Eppendorf (UKE), Hamburg, Germany

28 Institute for Health Economics and Healthcare Research, University Medical Center Hamburg-Eppendorf (UKE), Hamburg, Germany

29 Clinic of Ophthalmology, University Medical Center Hamburg-Eppendorf (UKE), Hamburg, Germany

30 Institute of Human Genetics, University Medical Center Hamburg-Eppendorf (UKE), Hamburg, Germany

31 Institute for Psychosomatic Medicine and Psychotherapy, University Medical Center Hamburg-Eppendorf (UKE), Hamburg, Germany

32 Department of Electrophysiology, Hamburg University Heart Center, University Heart and Vascular Center, Hamburg, Germany

33 Competence Center for Epidemiology and Health Services Research for Healthcare Professionals (CVcare), Institute for Health Services Research in Dermatology and Nursing (IVDP), University Medical Center Hamburg-Eppendorf (UKE), Hamburg, Germany

34 Institute for Tumor Biology, University Medical Center Hamburg-Eppendorf (UKE), Hamburg, Germany

35 Department of Pathology, University Medical Center Hamburg-Eppendorf (UKE), Hamburg, Germany

36 Department of General Practice and Primary Care, University Medical Center Hamburg-Eppendorf (UKE), Hamburg, Germany

37 Competence Center for Health Services Research in Dermatology (CVderm), Institute for Health Services Research in Dermatology and Nursing (IVDP), University Medical Center Hamburg-Eppendorf (UKE), Hamburg, Germany

38 Airway Research Center North, German Center for Lung Research, Grosshansdorf, Germany 